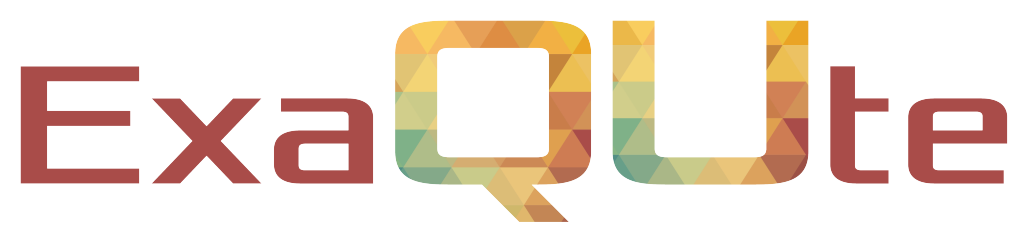

Exascale Quantification of Uncertainties for

Technology and Science Simulation

\title{
D1.4 Final public Release of the solver
}

\section{Document information table}

\begin{tabular}{|l|l|}
\hline Contract number: & 800898 \\
\hline Project acronym: & ExaQUte \\
\hline Project Coordinator: & CIMNE \\
\hline Document Responsible Partner: & CIMNE \\
\hline Deliverable Type: & Software release \\
\hline Dissemination Level: & Public \\
\hline Status: & Final version \\
\hline
\end{tabular}

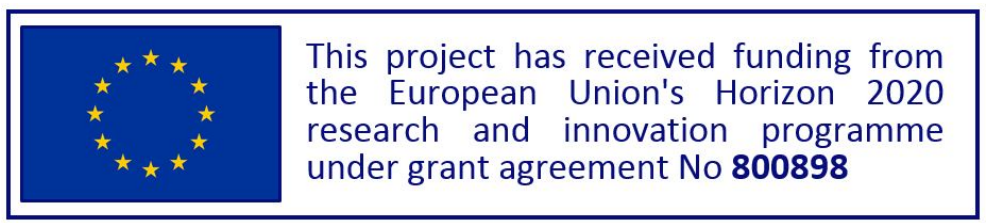




\section{Authoring}

\begin{tabular}{|c|c|c|c|c|}
\hline \multicolumn{5}{|l|}{ Prepared by: CIMNE } \\
\hline Authors & Partner & Modified & Version & Comments \\
\hline $\begin{array}{l}\text { Quentin Ayoul-Guilmard } \\
\text { Sundar Ganesh } \\
\text { Fabio Nobile }\end{array}$ & EPFL & \multirow{6}{*}{ All } & \multirow{6}{*}{ 1.0.0 } & $\mathrm{XMC}$ \\
\hline $\begin{array}{l}\text { Rosa M. Badia } \\
\text { Jorge Ejarque }\end{array}$ & $\mathrm{BSC}$ & & & PyCOMPSs \\
\hline $\begin{array}{l}\text { Luca Cirrottola } \\
\text { Algiane Froehly }\end{array}$ & INRIA & & & ParMmg \\
\hline $\begin{array}{l}\text { Brendan Keith } \\
\text { Anoop Kodakkal }\end{array}$ & TUM & & & Wind generator \\
\hline $\begin{array}{l}\text { Marc Nuñez } \\
\text { Carlos Roig } \\
\text { Riccardo Rossi } \\
\text { Riccardo Tosi }\end{array}$ & \multirow[t]{2}{*}{ CIMNE } & & & Kratos Multiphysics, XMC \\
\hline Cecilia Soriano & & & & Coordination \\
\hline
\end{tabular}

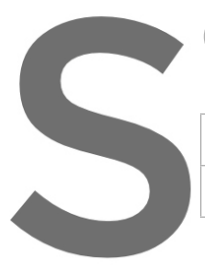

Change Iog

Versions Modifie

1.0 .0
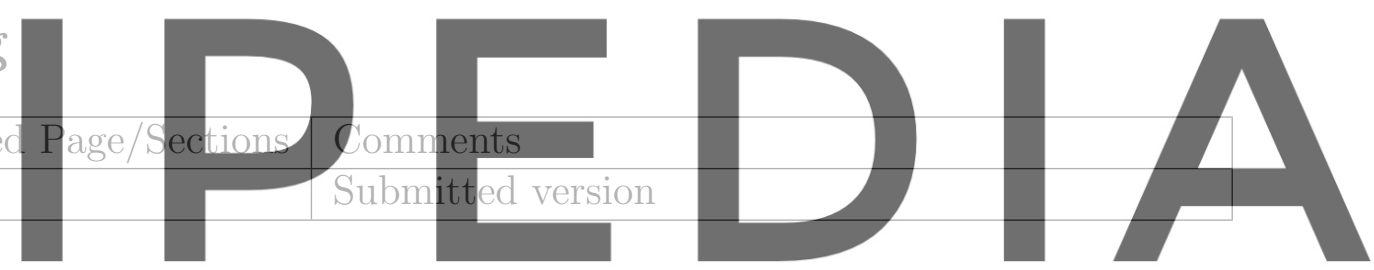

Approval

Register for free at https//www.scipedia.com to download the version without the watermark

Aproved by:

\begin{tabular}{|l|l|l|l|l|} 
& Name & Partner & Date & OK \\
\hline Task leader & Riccardo Rossi & CIMNE & $30 / 11 / 2020$ & OK \\
\hline WP leader & Riccardo Rossi & CIMNE & $30 / 11 / 2020$ & OK \\
\hline Coordinator & Riccardo Rossi & CIMNE & $30 / 11 / 2020$ & OK \\
\hline
\end{tabular}




\section{Executive summary}

This deliverable presents the final software release of Kratos Multiphysics, together with the XMC library, Hyperloom and PyCOMPSs API definitions [13]. This release also contains the latest developements on MPI parallel remeshing in ParMmg. This report is meant to serve as a supplement to the public release of the software.

Kratos is "a framework for building parallel, multi-disciplinary simulation software, aiming at modularity, extensibility, and high performance. Kratos is written in $\mathrm{C}++$, and counts with an extensive Python interface". XMC is "a Python library for parallel, adaptive, hierarchical Monte Carlo algorithms, aiming at reliability, modularity, extensibility and high performance". Hyperloom and PyCOMPSs are environments for enabling parallel and distributed computation. ParMmg is an open source software which offers the parallel mesh adaptation of three dimensional volume meshes.
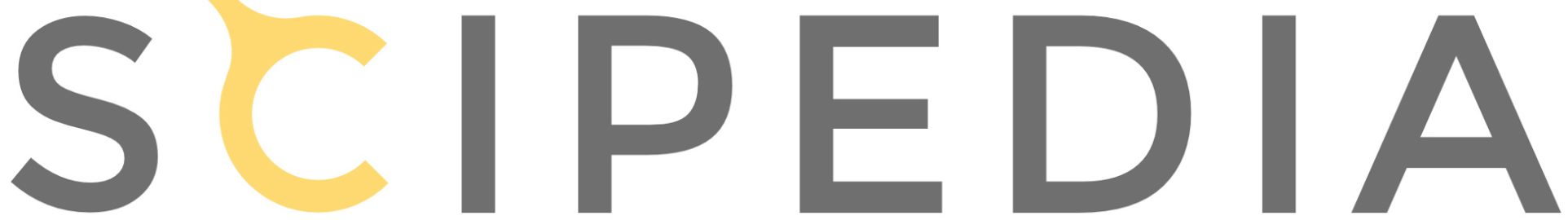

Register for free at https//www.scipedia.com to download the version without the watermark 


\section{Table of contents}

1 Introduction $\quad 6$

2 Software structure $\quad 6$

2.1 Multiphysics software . . . . . . . . . . . . . . . . 6

2.2 Uncertainty Quantification . . . . . . . . . . . . . . 7

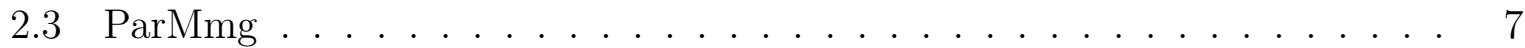

\section{Examples $\quad 8$}

3.1 Uncertainty Quantification and Ensemble Average . . . . . . . . . . . 8

3.2 MPI fluid dynamics simulations and parallel adaptive refinement with ParMmg .............................. 9
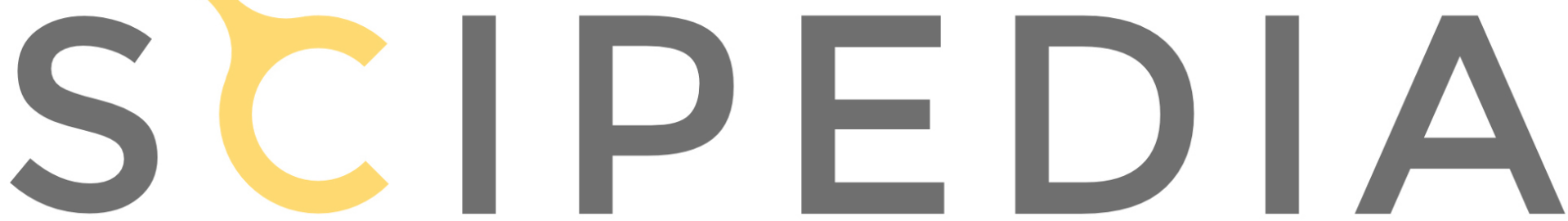

Register for free at https//www.scipedia.com to download the version without the watermark 


\section{Nomenclature / Acronym list}

\begin{tabular}{|l|l|}
\hline Acronym & Meaning \\
\hline API & Application Program Interface \\
\hline Kratos & Kratos MultiPhysics \\
\hline UQ & Uncertainty Quantification \\
\hline
\end{tabular}
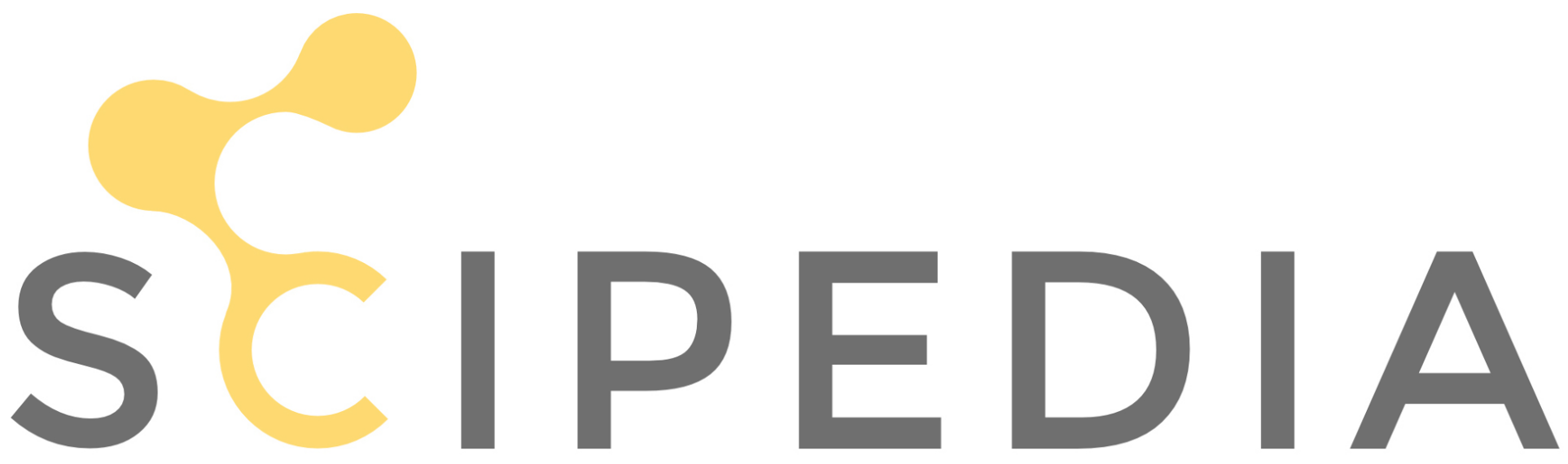

Register for free at https//www.scipedia.com to download the version without the watermark 


\section{Introduction}

The Kratos software [10, 11] is designed for dealing with a multitude of different physical problems, spacing from computational fluid dynamics to convection diffusion, from structural applications to fluid structure interaction. For more information, we refer to the Kratos documentation.

Kratos is mainly used in the project as the base multiphysics solver in each of the developments of the project. Kratos is designed in an structured manner by means of applications, which allows to develop and use a wide variety of numerical tools, methods and solvers in the same base framework. For the purpose of the project, the main application used as main solver is the FluidDynamicsApplication, a finite-element CFD framework. This application of Kratos can deal with incompressible Navier-Stokes problems on classical finite-element unstructured meshes and also on immersed meshes, which meets the requirements of the project considering the physical problem to be solved. Other applications will be used, which are detailed on section 2.1.

As explained in previous software release deliverables [1, 2, 4, 19], Kratos can solve Uncertainty Quantification problems. UQ is the field of science which studies how uncertainties evolve and propagate in problems, which are characterized by random parameters. UQ methods have been developed within the XMC Python library [3], and Kratos is the default solver software of the in $[1,4]$.
Both XMC and Kratos are designed fo
ronments. The scheduling in distributed en
Hyperloom [8] and PyCOMPS $[5,15,18]$. X libraries. Due to this inner integration of
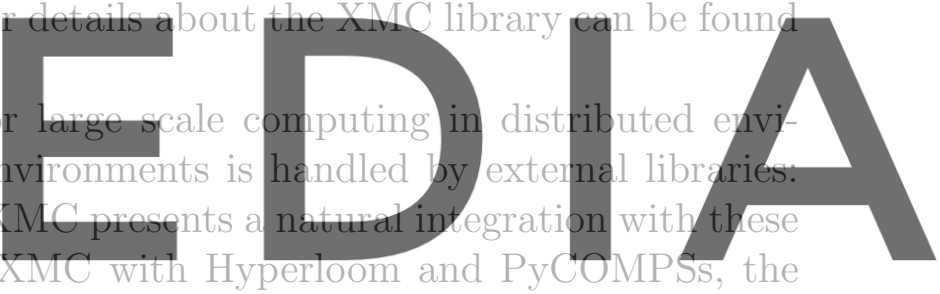

API definitions of the schedulers have been integrated in the software release.

Register for free at https/ywww scipedia. com to download the versipr without the watermark software developed by INRIA: ParMmg. An interface with Kratos has been developed, and it enables the use of the remeshing software along with the multiphysics solvers.

Therefore, this final software release [13] contains Kratos, XMC, ParMmg, Hyperloom and PyCOMPSs API definitions. The complete changelog is available in the Kratos releases page.

\section{Software structure}

\subsection{Multiphysics software}

Kratos Multiphysics 8.1 release can be retrieved from the Kratos releases page. The main applications that are used in the project are:

- ExaquteSandboxApplication: Contains custom processes and utilities used to run the examples in the ExaQUte project.

- FluidDynamicsApplication: Contains all CFD formulations and utilites, including body-fitted and embedded incompressible Navier-Stokes solvers.

- LinearSolversApplication: Contains a wide selection of different linear solvers (LU, multi-grid, eigen-solvers) to solve the linear equation on the FEM analysis. 
- MappingApplication: contains the core developments in mapping data between non matching grids. For instance, two different meshes with different discretization.

- MeshingApplication: Contains several utilities to perform meshing and remeshing, as well as the wrapper to use Mmg and ParMmg.

- MetisApplication: Wrapper for Metis library to be used within Kratos.

- MultilevelMontecarloApplication: Contains a wrapper with the XMC library and utilites to perform UQ.

- StatisticsApplication: Contains utilities to compute statistical measures of the physical quantites involved in the analyses.

- Trilinos Application: Wrapper for Trilinos library to be used within Kratos.

\subsection{Uncertainty Quantification}

Within Kratos, different applications are organized in different folders. As described in previous works $[2,19]$, one folder is entirely dedicated to UQ and it is called MultilevelMonteCarloApplication. The folder is located in:

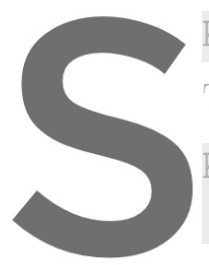
Kratos/applicatic This folder contains Kratos/applicationi $\mathrm{XMC}$

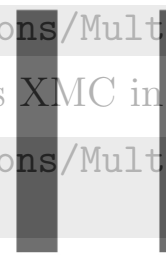

and Hyperloom and PyCOMPSs API definitions in:
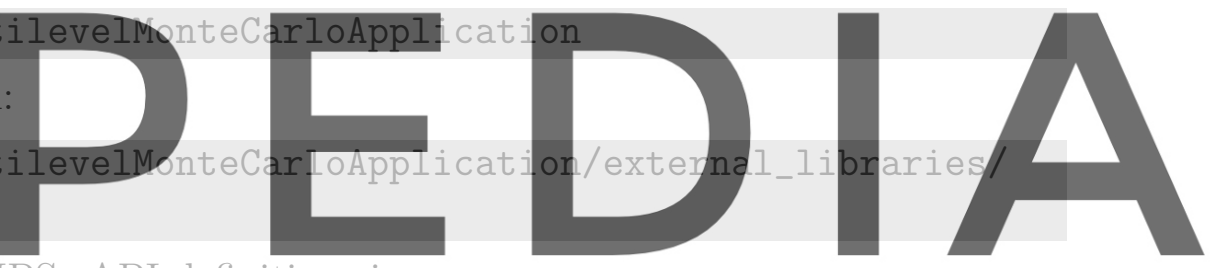

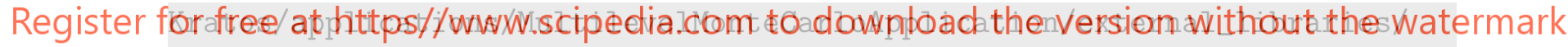
PyCOMPSis/exaqute

\subsection{ParMmg}

ParMmg is the MPI parallel version of the metric-based refinement sofware Mmg. Mmg is an open source software for bidimensional and tridimensional surface and volume remeshing. For this release, ParMmg offers the parallel mesh adaptation of three dimensional volume meshes $[6,9,12]$.

In this release of the solvers, an interface in Kratos with the recent developments on the MPI parallel remeshing software ParMmg is included. This allows to perform parallel refinement operations from the Kratos solver without effort from the user, as the interface contains the library calls to ParMmg and takes care on adapting the data structures from Kratos to ParMmg.

These remeshers require the definition of a metric field, which will set the desired size and orientation of the final elements on the remeshed mesh. A metric is a tensor of size $d \mathrm{x} d$ where $\mathrm{d}$ is the number of dimensions. The computation of the metrics is available in the MeshingApplication of Kratos.

The releases of Mmg and ParMmg can be downloaded from: 


\section{Examples}

Kratos examples has been released together with the software release, and can be found here [14]. In the following sections, examples on UQ and adaptive refinement will be presented, which will also serve as an example of usage of Kratos Multiphysics, with examples directly related to the project.

\subsection{Uncertainty Quantification and Ensemble Average}

Different algorithms have been developed during the project within the XMC library: Monte Carlo, Multilevel Monte Carlo, Adaptive Monte Carlo, Adaptive Multilevel Monte Carlo, Continuation Multilevel Monte Carlo, Asynchronous Monte Carlo and Asynchronous Multilevel Monte Carlo. We refer to previous works for details [1, 2, 4, 19$]$.

In addition to previous deliverable, XMC can be exploited as well to solve unsteady and ergodic problems, applying the ensemble average method [16].

Referring to the Examples release [14], all problems are located in the

Examples/multilevel_monte_carlo

folder and they are organized in two subfolders: validation and use_cases. The former validates the software

that were solved in

sory Aeronautical C

many folders as problems th

user can easily underst

both.
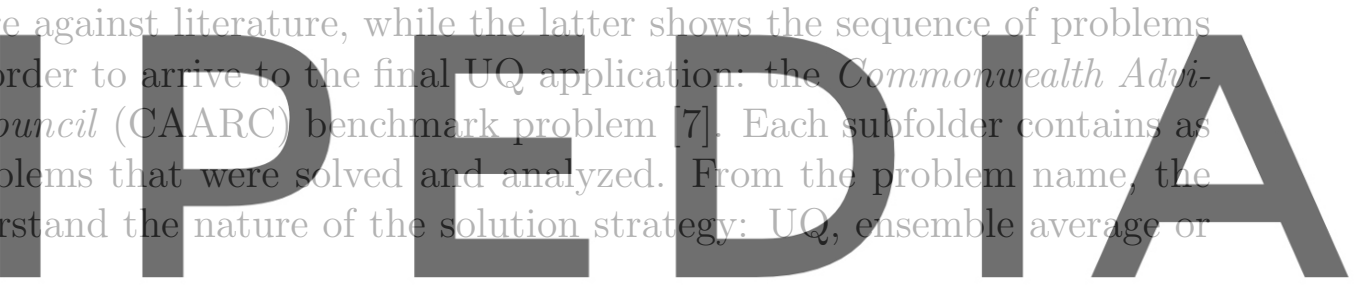

Problem descriptions and instructions to run each problem can be found within each

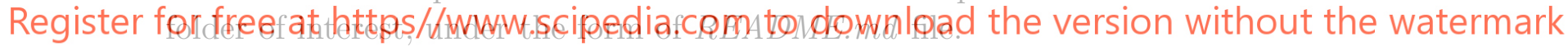

By default, all problems run in serial. In case the user is interested in running in parallel, in every folder bash files are added. These can be used as a reference to run using Hyperloom or PyCOMPSs. Moreover, in each execution script ${ }^{1}$ and in the XMC file

\section{Kratos/applications/MultilevelMonteCarloApplication/external_libraries/ \\ $\mathrm{XMC} / \mathrm{xmc} /$ distributedEnvironmentFramework.py}

the appropriate import has to be changed from

from exaqute.ExaquteTaskLocal import *

to

from exaqute.ExaquteTaskHyperLoom import *

or

from exaqute.ExaquteTaskPyCOMPSs import *

For further details about running in distributed environments, we refer to the MultilevelMonteCarloApplication documentation and to the PyCOMPSs and Kratos tutorial.

\footnotetext{
${ }^{1}$ Execution scripts are called run_mc_Kratos.py or run_mlmc_Kratos.py.
} 


\subsection{MPI fluid dynamics simulations and parallel adaptive re- finement with ParMmg}

Prior to this release, the remesher Mmg was already available, and it was covered in the previous Deliverable 1.3 [2]. It is reminded that there is a set of examples for the serial remesher, showcasing the definition of different metrics and the use of Mmg software in the Kratos Examples release [14], located in:

\section{Examples/mmg_remeshing_examples}

On this release, a new set of examples is added with the recent developments on ParMmg, which are located in:

\section{Examples/parmmg_remeshing_examples}

These examples can only be run in MPI. The first example is a basic fluid dynamics channel flow with a body-fitted cylinder. The script solves a few seconds of simulation, and then the information on the velocity and pressure field is used to adaptively refine the mesh. This process is repeated several times, improving gradually the accuracy on the solution. This is an example of how the solution of a previous simulation can be used to improve future simulations. It is also a showcase of how all involved operations can be performed in parallel: the physics simulation, the metrics computation, the remeshing operation and the map
are located in the $R E A$
The second example
related to the project.
building is loaded by
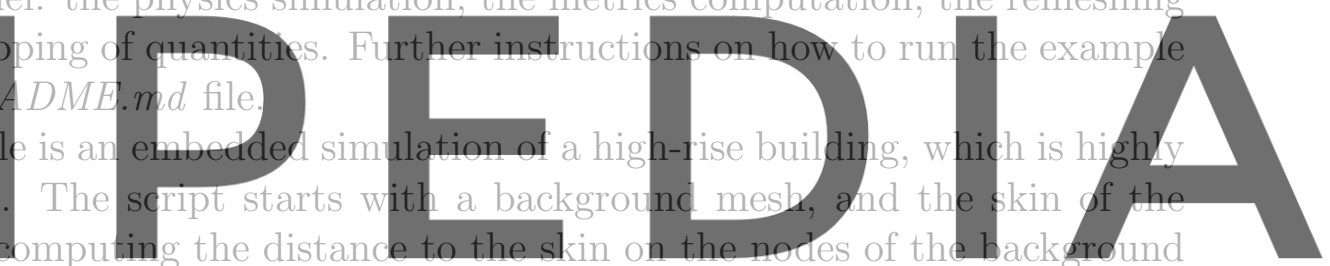

mesh. The information of this distance can be used as well to refine adaptively. The

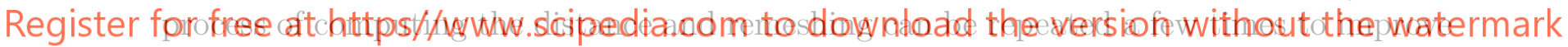

the resolution of the geometry. After finishing the remeshing process, the embedded

simulation is started on the improved background mesh. In this case, the remeshing is performed before starting the fluid dynamics simulation as a pre-process. This is also a perfect showcase of what would be needed in an optimization loop, as every optimization step modifies slightly the geometry. In each optimization step, the mesh could be adapted to the new design to keep an accurate representation of the geometry. Further instruction on how to run the example are located in the README.md file.

\section{Current results}

The work developed and released has been run and tested with several benchmarks, which have been run in different supercomputers. The software has been tested up to 192 working nodes (9216 CPUs), and showed a very good parallel efficiency. Part of the work has already been submitted to a peer reviewed journal, while other works will be submitted soon.

Regarding the adaptive refinement strategies, some figures are presented next. Firstly, Figure 4.4 shows the body-fitted cylinder case, which was run with 32 MPI processors. It uses the information of the velocity field at every time step to perform the refinement. With this example, the user can test other combinations of physical quantities such as the pressure, to get a different adaptation. Next, Figures 4.5 and 4.6 show a before-after 


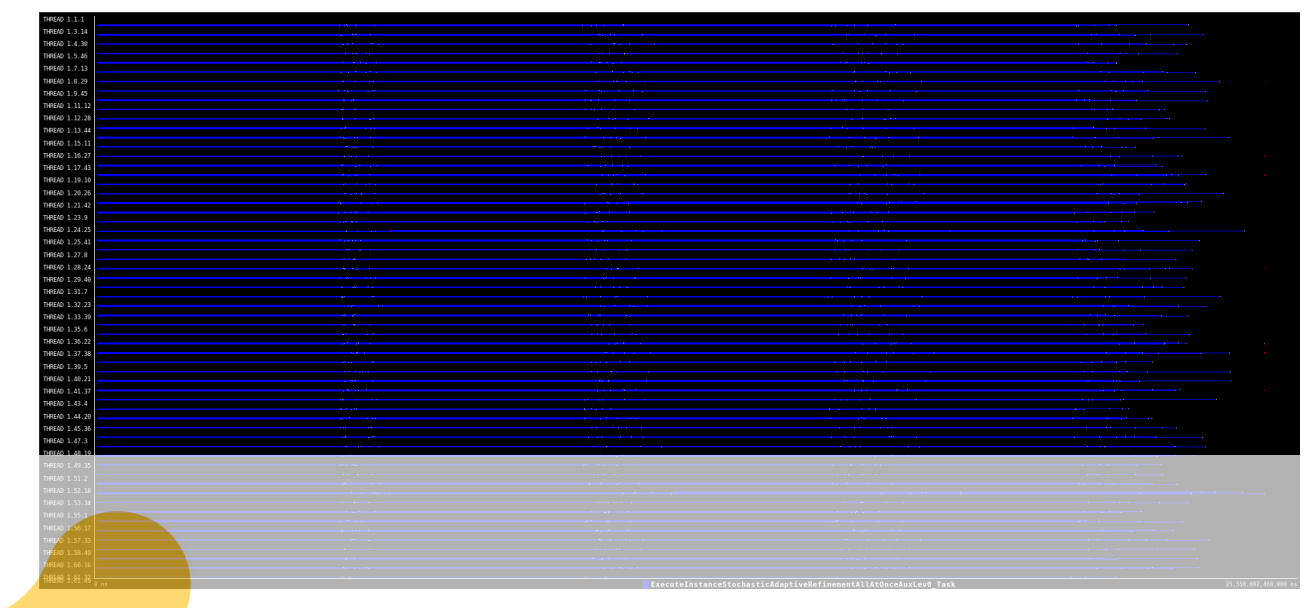

Figure 4.1: Execution trace of asynchronous Monte Carlo algorithm, running with PyCOMPSs with 41 worker nodes / 1968 CPUs. Blue lines are job executions, and they completely fill the machine during the whole runtime. The problem is the wind

engineering two-dimensional benchmark (see [14] for details).
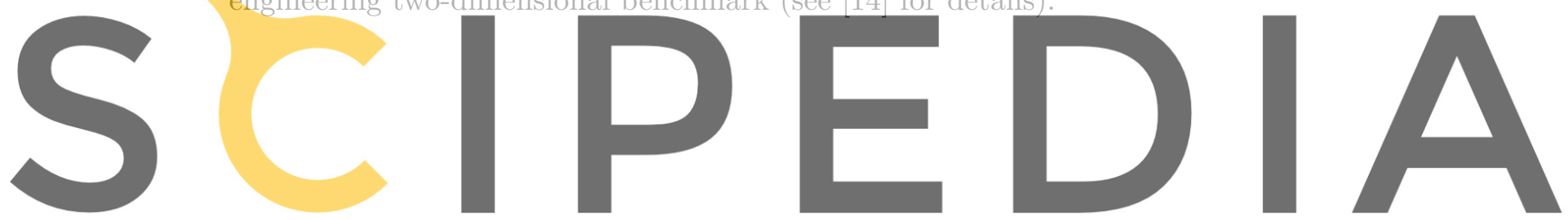

Register for free at https//www.scipedia.com to download the version without the watermark

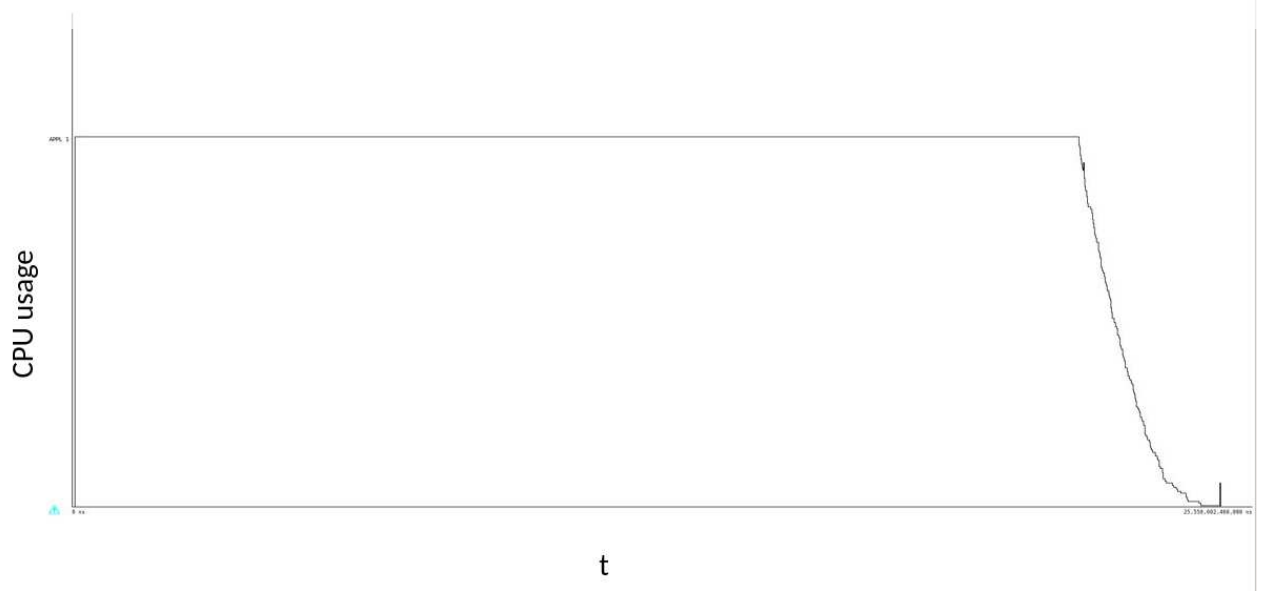

Figure 4.2: CPU usage of asynchronous Monte Carlo algorithm, running with PyCOMPSs with 41 worker nodes / 1968 CPUs. CPU usage is constant and equal to $100 \%$ of the machine during the whole runtime. The problem is the wind engineering two-dimensional benchmark (see [14] for details). 


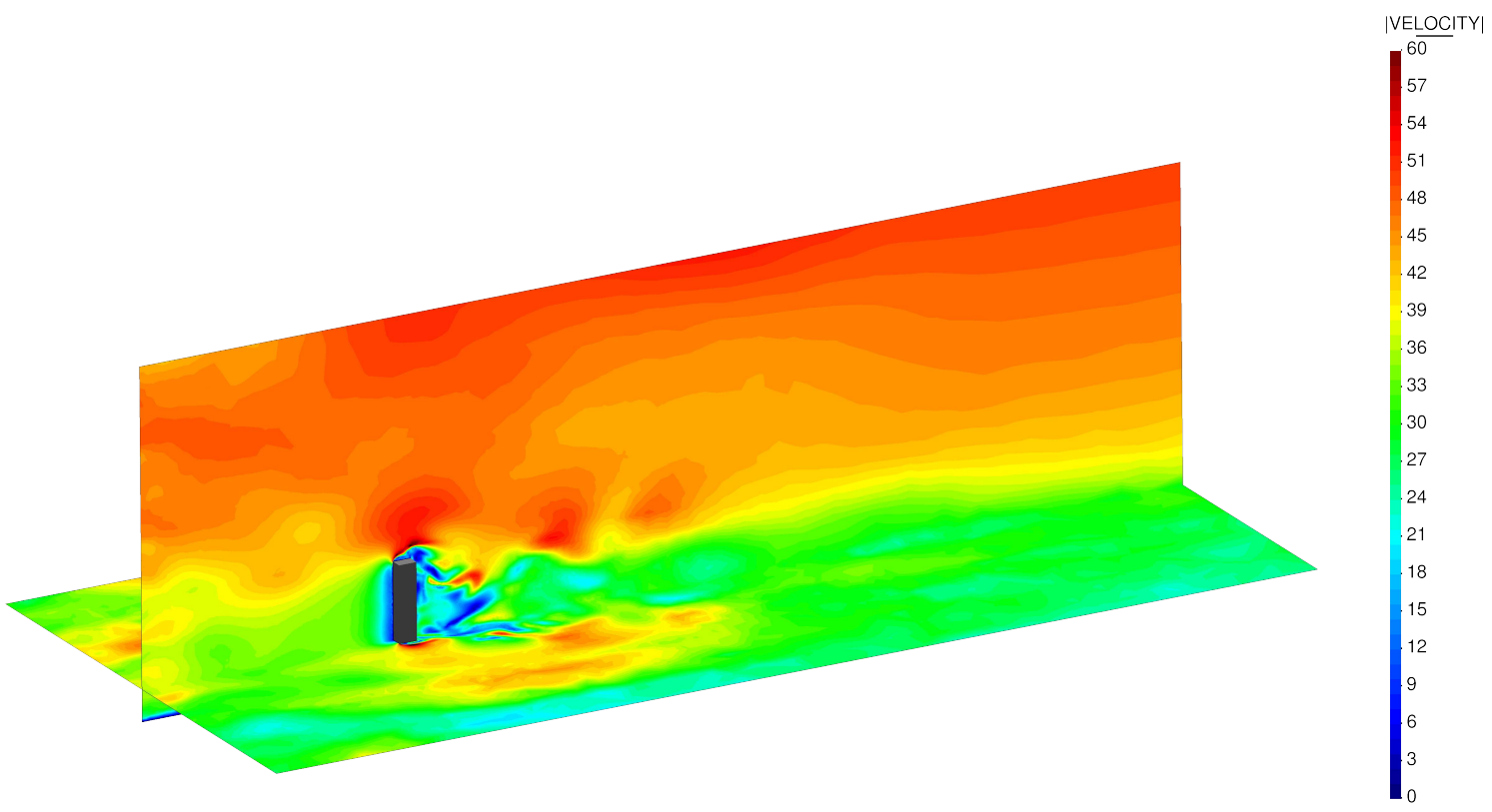

Figure 4.3: Velocity field around the CAARC building. The problem has been run with the Mann wind model [17].

comparison of an adaptive refinement strategy using the distance field with 256 MPI processors. It can be seen that discretization is not good enough to correctly represent the building in the initial mesh. Adapting the mesh improves the description of the building, which is then used to start an embedded fluid dynamic analysis of the incompressible Navier-Stokes flow around the building. A snapshot of the velocity field in the embedded simulation on the remeshed mesh is shown in Figure 4.7.

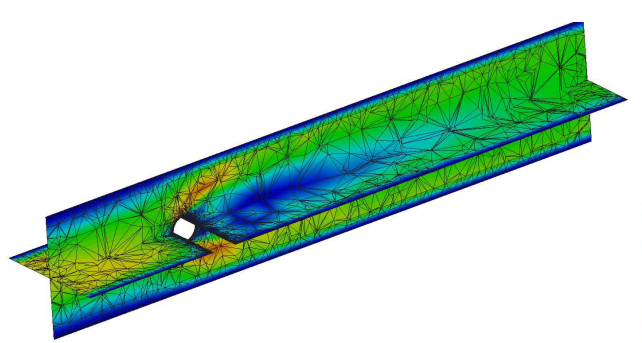

(a) Initial mesh and its velocity field at $\mathrm{T}=5.0$ s with 100k elements.

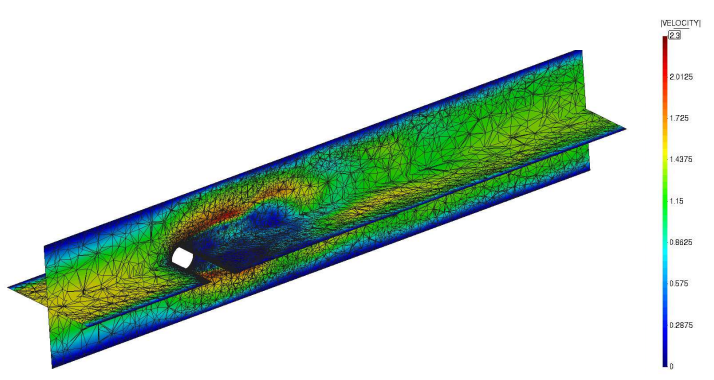

(b) Remeshed mesh at the fourth iteration using the velocity from previous solutions at $\mathrm{T}=5.0 \mathrm{~s}$ with $700 \mathrm{k}$ elements.

Figure 4.4: Comparison of the initial and final mesh after four iterations on a consecutive resolution and refinement process of a body-fitted cylinder mesh. 

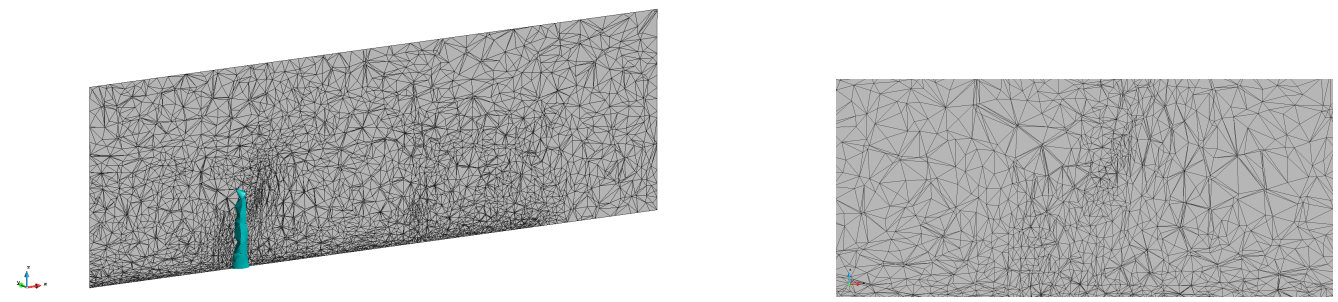

(a) Vertical cut and isosurface representation (b) Zoom on the mesh where the building is of a high-rise building using a distance function located. If compared to Figure 4.6 (b), it can be on an unadapted mesh. It can be seen that the seen that the nodal presence is not enough to resolution of the mesh is not enough to properly obtain a proper representation of the building account for the geometry.

Figure 4.5: Vertical cuts on an unadapted background mesh with 200k elements where a high-rise building is loaded with a distance function. This mesh serves as the initial ground to load the geometry, and it is therefore not utilized to solve the fluid dynamics problem.

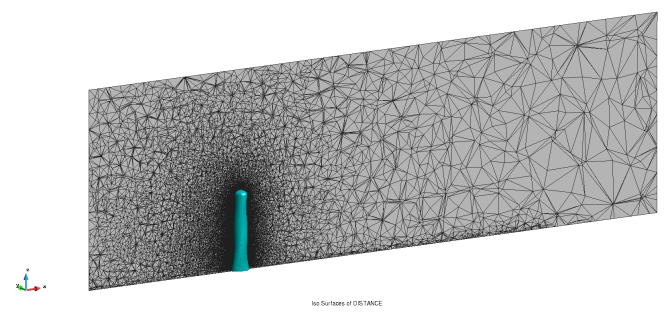

(a) Vertical cut and isosurface representation of (b) Zoom on the mesh where the building is a high-rise building using a distance function on located. Since the metric was built according an adapted mesh. Adapting the mesh increases to a distance function representing the buildthe

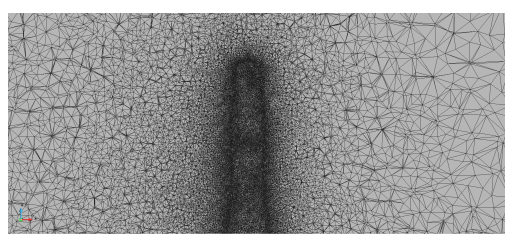
ing, smaller elements are accomplished near the skin, where the distance is close to 0 .

Figure 4.6: Vertical cuts on an adapted background mesh with $2 \mathrm{M}$ elements where a high-rise building is loaded with a distance function. An embedded solver is used to solve the incompressible Navier-Stoke flow around the building. The refinement was performed with 256 MPI processors. 


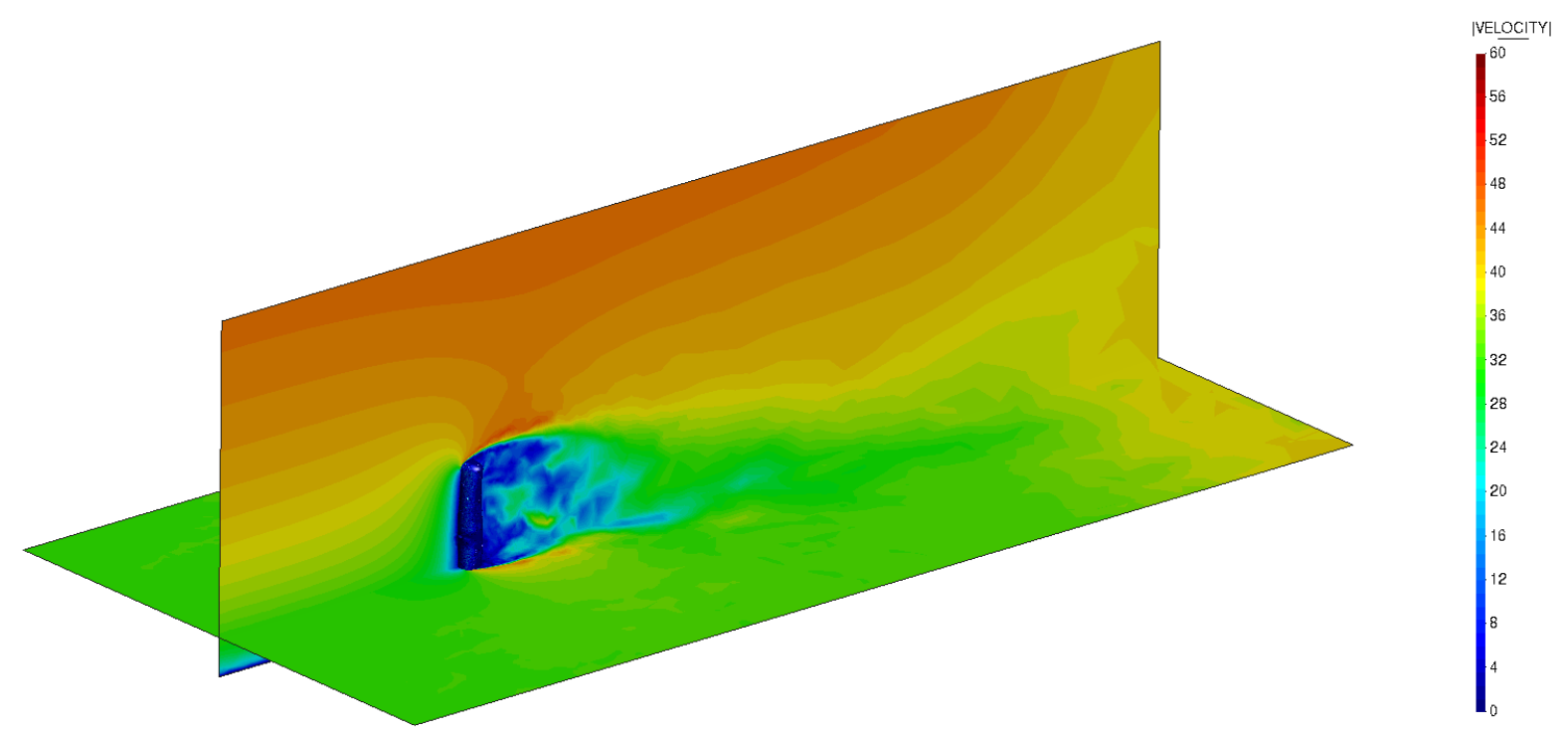

Figure 4.7: Velocity field around the embedded building with a steady logarithmic wind inlet. The geometry is represented by a distance function and the problem is solved using an embedded solver after remeshing with ParMmg. The analysis was run with 256 MPI processors.

\section{References}

[1] R. Amela, Q. Ayoul-Guilmard, R. M. Badia, S. Ganesh, F. Nobile, R. Rossi, and R. Tosi. D5.2 Release of ExaQUte MLMC Python engine, May 2019.

[2] Q. Ayoul-Guilmard, R. M. Badia, J. Ejarque, M. Núñez, S. Ganesh, F. Nobile, C. Soriano, C. Roig, R. Rossi, and R. Tosi. D1.3 First public Release of the solver. Technical report, BSC, CIMNE, EPFL, 2020.

[3] Q. Ayoul-Guilmard, S. Ganesh, F. Nobile, R. Rossi, R. Tosi, R. M. Badia, and R. Amela. XMC, 2020. URL https://doi.org/10.5281/zenodo. 3235832.

[4] Q. Ayoul-Guilmard, M. Núñez, S. Ganesh, F. Nobile, R. Rossi, and R. Tosi. D5.4 Report on MLMC for time dependent problems. Technical report, ExaQUte, 2020.

[5] R. M. Badia, J. Conejero, C. Diaz, J. Ejarque, D. Lezzi, F. Lordan, C. Ramon-Cortes, and R. Sirvent. COMP superscalar, an interoperable programming framework. SoftwareX, 3-4, 12 2015. doi:10.1016/j.softx.2015.10.004.

[6] P. Benard, G. Balarac, V. Moureau, C. Dobrzynski, G. Lartigue, and Y. D'Angelo. Mesh adaptation for large-eddy simulations in complex geometries. International Journal for Numerical Methods in Fluids, 81(12):719-740, 2016. doi:https://doi.org/10.1002/fld.4204. URL https://onlinelibrary.wiley.com/ $\mathrm{doi} / \mathrm{abs} / 10.1002 / \mathrm{fld} .4204$.

[7] A. L. Braun and A. M. Awruch. Aerodynamic and aeroelastic analyses on the CAARC standard tall building model using numerical simulation. Computers and Structures, 87(9-10):564-581, may 2009. ISSN 
00457949. doi:10.1016/j.compstruc.2009.02.002. URL http://dx.doi.org/10. 1016/j.compstruc. 2009.02.002.

[8] V. Cima, S. Böhm, J. Martinovič, J. Dvorskỳ, K. Janurová, T. V. Aa, T. J. Ashby, and V. Chupakhin. Hyperloom: A platform for defining and executing scientific pipelines in distributed environments. In Proceedings of the 9th Workshop and 7th Workshop on Parallel Programming and RunTime Management Techniques for Manycore Architectures and Design Tools and Architectures for Multicore Embedded Computing Platforms, pages 1-6. ACM, 2018.

[9] L. Cirrottola and A. Froehly. Parallel unstructured mesh adaptation using iterative remeshing and repartitioning. Research Report RR-9307, INRIA Bordeaux, équipe CARDAMOM, Nov. 2019. URL https://hal.inria.fr/hal-02386837.

[10] P. Dadvand, R. Rossi, and E. Oñate. An object-oriented environment for developing finite element codes for multi-disciplinary applications. Archives of computational methods in engineering, 17(3):253-297, 2010.

[11] P. Dadvand, R. Rossi, M. Gil, X. Martorell, J. Cotela, E. Juanpere, S. R. Idelsohn, and E. Oñate. Migration of a generic multi-physics framework to HPC environments. Computers and Fluids, 80(1):301-309, 2013. ISSN 00457930. doi:10.1016/j.compfluid.2012.02.004.

[12] C. Dapogny, C. Dobrzynski, and P. Frey. Three-dimensional adaptive domain remeshing, implicit domain meshing, and applications to free and moving boundary problems. Journal of Computational Physics, 262:358 - 378, 2014. ISSN 0021-9991. doi:https://doi.org/10.1016/j.jcp.2014.01.005. URL http://www.sciencedirect. com/science/article/pii/S0021999114000266.

[13] V. M. Ferrándiz, P. Bucher, R. Rossi, jcotela, J. Maria, R. Zorrilla, M. A. Celigueta, G. Casas, C. Roig, A. C. Velázquez, P. Dadvand, S. Latorre, J. I. González, I. de Pouplana, miguelmaso, M. Núñez, F. Arrufat, dbaumgaertner, B. Chandra, A. Ghantasala, armingeiser, S. Warnakulasuriya, lluís, J. Gárate, MFusseder, Pablo, AFranci, L. Gracia, thomas, K. B. Sautter, and R. Tosi. KratosMultiphysics/Kratos: Release 8.1, 2020. URL https://doi.org/10.5281/zenodo.3234644.

[14] V. M. Ferrándiz, R. Tosi, I. de Pouplana, R. Zorrilla, L. Gracia, S. Warnakulasuriya, M. Núñez, B. Chandra, A. Ghantasala, P. Bucher, jcotela, AFranci, F. Arrufat, K. B. Sautter, S. Latorre, jgonzalezusua, A. Geiser, P. Dadvand, miguelmaso, dbaumgaertner, M. A. Celigueta, RahulKN, swenczowski, BasselSaridar, C. Roig, M. Zidan, and G. Casas. KratosMultiphysics/Examples: Kratos Examples 8.1, Nov. 2020. URL https://doi.org/10.5281/zenodo. 4293799.

[15] F. Lordan, E. Tejedor, J. Ejarque, R. Rafanell, J. Álvarez, F. Marozzo, D. Lezzi, R. Sirvent, D. Talia, and R. M. Badia. ServiceSs: An Interoperable Programming Framework for the Cloud. Journal of Grid Computing, 12(1):67-91, 2014. ISSN 15707873. doi:10.1007/s10723-013-9272-5.

[16] V. Makarashvili, E. Merzari, A. Obabko, A. Siegel, and P. Fischer. A performance analysis of ensemble averaging for high fidelity turbulence simulations at the 
strong scaling limit. Computer Physics Communications, 2017. ISSN 00104655. doi:10.1016/j.cpc.2017.05.023.

[17] J. Mann. Wind field simulation. Probabilistic Engineering Mechanics, 13(4):269-282, 1998. ISSN 02668920. doi:10.1016/s0266-8920(97)00036-2.

[18] E. Tejedor, Y. Becerra, G. Alomar, A. Queralt, R. M. Badia, J. Torres, T. Cortes, and J. Labarta. PyCOMPSs: Parallel computational workflows in Python. International Journal of High Performance Computing Applications, 31(1):66-82, 2017. ISSN 17412846. doi:10.1177/1094342015594678.

[19] R. Tosi, R. Amela, R. Badia, M. Núñez, and R. Rossi. D1.2 First release of the softwares. Technical report, BSC, CIMNE, 2019. 